\title{
Quality of life and mortality after surgical treatment for vertebral osteomyelitis (VO): a prospective study
}

\author{
Ayla Yagdiran ${ }^{1}$ (1) $\cdot$ Christina Otto-Lambertz ${ }^{1} \cdot$ Kristina Michaela Lingscheid $^{1} \cdot$ Krishnan Sircar $^{1}\left(\mathbb{D} \cdot\right.$ Christina Samel $^{2}$.

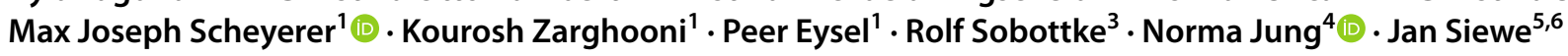

Received: 4 February 2020 / Revised: 2 May 2020 / Accepted: 20 June 2020 / Published online: 1 July 2020

(c) The Author(s) 2020

\begin{abstract}
Purpose Vertebral osteomyelitis (VO) has a high mortality and leads to chronic pain and functional disability. Surgical treatment is often necessary. To date, little is known about the consequences of surgery on patient outcome. The aim of this study was to determine the quality of life (QoL) and mortality rates of surgically treated VO patients for a period of 2 years. Methods Patients with VO undergoing surgical treatment in a tertiary referral hospital from 2008 to 2015 were included prospectively. Data were collected before (T0) as well as 1 year (T1) and 2 years (T2) post-surgery. Within the European Spine Tango registry, prospective patient and QoL data were collected using validated outcome scores: Oswestry Disability Index, Short Form 36/EuroQol, Visual Analog Scale, and Core Outcome Measures Index.

Results From 195 patients surgically treated for VO, QoL data were available from 136 patients at $T 0,100$ patients at $T 1$, and 82 patients at $T 2$, respectively. The 1- and 2-year mortality rates were $20 \%$ and $23 \%$. Mainly all QoL outcome scores showed significant improvement at $T 1$ and did not change significantly from $T 1$ to $T 2$.

Conclusion Surgical treatment of VO patients leads to significantly improved QoL. Nevertheless, QoL levels were below those of the general population. Our results underscore that spine disability questionnaires measuring QoL are mandatory to demonstrate comprehensively the severity of this entity. Our study confirms a high mortality and points out the role of $\mathrm{VO}$ as a potentially life-threatening condition.
\end{abstract}

Keywords Spondylodiscitis · Oswestry Disability Index (ODI) · Short Form 36 (SF-36) · Visual Analogue Scale (VAS) • Core Outcome Measures Index (COMI)

\section{Introduction}

With 5-6 new cases per 100,000 population per year, an increase in vertebral osteomyelitis (VO) could be observed in recent years. This is mainly attributed to improved diagnostics and demographic development showing an aging population with chronic debilitating diseases [1-6]. In 2015, the Federal Statistical Office of Germany determined an incidence of 30 new cases per 250,000 population per year [7]. The overall mortality has been reported up to $20 \%$ and appears to be particularly high in the first year after

Ayla Yagdiran is the first author. Norma Jung and Jan Siewe contributed equally to the work and therefore share the senior authorship.

Ayla Yagdiran

ayla.yagdiran@uk-koeln.de

Extended author information available on the last page of the article diagnosis $[5,8,9]$. Because the diagnosis is often delayed, $\mathrm{VO}$ is frequently associated with chronic pain and loss of mobility and function $[10,11]$. The principal treatment for VO is targeted antibiotic therapy with or without surgery [3, 4]. Surgery is indicated in cases of instability of the affected spinal segment, failure of conservative therapy, and a progressive neurological deficit $[6,12]$. There have only been a few studies regarding quality of life (QoL) in VO. Available data are based mainly on retrospective analyses with small case numbers [6, 12-19]. In the first 6 months, surgically treated patients seem to have better QoL than those conservatively treated [14]. Nevertheless, the QoL for VO patients is below that of the general population $[6,13,17]$. The goal of this study is the prospective evaluation of mortality and QoL data for VO patients surgically treated over a 2-year time period. In addition, these QoL data will be compared to QoL data of the general population given in the literature. 


\section{Materials and methods}

\section{Patient selection}

From January 2008 until January 2015, all inpatients of a tertiary referral hospital with suspected VO were screened for the study. Study inclusion criteria were the presence of characteristic back and/or leg pain confirmed by characteristic magnetic resonance imaging (MRI) or an abscess or vertebral body destruction detected by computed tomography (CT). Patients without indications for surgery were excluded.

\section{Data collection}

All data were prospectively collected with the help of the European "Spine Tango" register. To assess QoL, the following patient-related outcome measures (PROMs) were used: the Core Outcome Measures Index (COMI), the Oswestry Disability Index (ODI) and the Short Form 36 (SF-36) with physical and mental components (PCS and MCS) that was replaced in 2012 by the European Quality of Life Questionnaire (EQ-5D). All questionnaires are established and validated measures of QoL. The COMI consists of six items including two visual analogue scales (VASs), measuring back pain and leg pain, that result in a score from 0 to 10 ; the ODI consists of 10 items that result in a score from 0 to 100 . In both questionnaires, a lower average score demonstrates a higher QoL. The SF-36 consists of 36 items representing 8 dimensions including PCS and MCS that result in a score from 0 to 100, where a higher score demonstrates a higher QoL. The EQ-5D consists of 5 questions that result in a score from -0.6 to 1.0 , where a higher score demonstrates a higher QoL. The QoL data were collected preoperatively (T0) as well as after 12 (T1) and $24(T 2)$ months. Evaluation of patient data from "Spine Tango" was given ethical approval by the Faculty of Medicine at the University of Cologne (09-182). In addition, the following variables were recorded for all VO patients undergoing surgery: age, sex, duration of hospital stay, microbiological diagnosis, affected spine segment, preoperative neurological deficits, postoperative recurrence and comorbidity according to the American Society of Anesthesiologists (ASA) score. The (ASA) Physical Status Classification System was developed in 1941 to classify patient comorbidity and is widely used by clinicians. ASA Class I is defined as a normal healthy patient, and Class $\mathrm{V}$ as a moribund patient not expected to survive without surgery.

In addition to epidemiological and clinical parameters, mortality (at 1 and 2 years) and quality of life data over the 2-year observation period were collected.

\section{Surgical procedures}

The decision between surgical and non-surgical treatment was determined for each patient on defined criteria, as described below. Generally, patients without bony destruction of the endplates, suspected instability and intraspinal empyema were treated conservatively. Main indication for surgery was neurological impairment. In patients with progressive neurological failures with or without impending sepsis or hemodynamic instability, surgical intervention was performed immediately. Further indications were pain caused by spinal instability, progressive deformity and/or intraspinal empyema, or failure to respond to conservative antimicrobial therapy. Surgical treatment was performed according to the vertebral destruction. If only an abscess or even an empyema was present, intraspinal debridement was performed. In cases of bony destruction and/or instability, additional intercorporal fusion was carried out. If a reconstruction of the ventral column was necessary but not possible from the dorsal approach due to the extent of the bony destruction, a two-staged treatment was chosen: debridement and instrumentation in the first surgery and corporectomy/spondylodesis with implantation of iliac crest grafts, intervertebral cages or vertebral body replacement in the second surgery (ventral approach).

\section{Case verification}

All cases were discussed interdisciplinary between an infectious disease's specialist and an orthopedic surgeon to confirm the diagnosis of VO. The diagnosis of VO was rejected in patients without previous antimicrobial treatment, if the C-reactive protein was normal at presentation and no plausible pathogen could be identified. These patients and patients with a more plausible alternative diagnosis from histopathological findings (e.g., tumor, erosive osteochondrosis) were excluded from the analysis. The relevance and plausibility of the identified pathogens were separately verified by an infectious disease's specialist. A detected virulent organism such as Staphylococcus aureus and gram-negative bacteria in $\geq$ one sample or a detected low-virulent organism such as coagulase-negative staphylococci or Propionibacterium Species in at least two relevant samples was considered as the etiologic pathogen.

\section{Antibiotic treatment}

Except for patients with severe sepsis, no empirical antiinfective therapy was recommended preoperatively to identify the specimen. Whenever possible, antimicrobial therapy of VO was directed against the identified specimen. In culturenegative cases, empirical therapy was administered with our 
local standard regimen (ceftriaxone and flucloxacillin) as MRSA rates are low in Germany. In general, postoperative antibiotic treatment was administered intravenously for 14 days followed by a highly bioavailable oral antimicrobial therapy oralization with a duration of 6 to 12 weeks in total depending on the severity of diseases deferred response or risk factors (e.g., foreign material-associated infection). A longer intravenously therapy was applied when indicated (e.g., concomitant endocarditis; Staphylococcus aureus bacteremia; disseminated infectious complications). Therapy for specific microorganisms was applied according to the guidelines (e.g., Mycobacterium spp., Candida spp., Brucella spp.). The response to therapy was controlled clinically (pain reduction) and by laboratory inflammation marker (significant CRP drop). Control MRIs were only initiated with unfavorable treatment response (e.g., persistent back pain and/or high levels of CRP).

\section{Statistical analysis}

Counts and frequencies were used to describe the sample. To compare QoL at different time points, the Friedman test as well as post hoc Wilcoxon tests was performed. The survival curve was estimated with the Kaplan-Meier method. All statistical operations are of an exploratory nature; therefore, no adjustments were made for multiple testing. The significance level was set at 5\% for all reported inferential statistical operations. Data analyses and graphical depictions were performed using IBM SPSS Statistics Version 25 and R Version 3.5.1 ("Feather Spray").

\section{Results}

\section{Baseline characteristics}

From January 2008 until January 2015, 245 inpatients with confirmed VO were treated in our hospital, 195 of whom underwent surgery. In 136 cases, additional PROMs were collected (Fig. 1).

The 195 surgically treated VO patients had a median age of 69 years, and the majority were male $(66 \%)$. The lumbar spine was most commonly affected (56\%). $60 \%$ of the patients had severe comorbidities (ASA score $\geq 3$ ). Microbiological diagnosis was established in 138 patients (71\%). Staphylococcus aureus was the most commonly isolated pathogen $(n=68 / 138 ; 49 \%)$. The recurrence rate was low (4\%). Neurologic impairment at diagnosis occurred in 57 patients (29\%). Mean hospital stay was 33 days (Table 1).

\section{Mortality}

Thirty-eight patients died within 12 months, and 45 patients within 24 months. This yields 1 - and 2-year mortality rates of $20 \%$ and $23 \%$, respectively (Fig. 2).

\section{Quality of life}

The follow-up results of the individual QoL scores are listed in Table 2.

Self-reported VAS scores for back and leg pain decreased significantly in the first 12 months (T1) and remained nearly constant in the following 12 months (T2); see Fig. 3.

QoL showed similar results. ODI, SF-36 PCS and EQ-5D showed significant improvements at $T 1$ vs. $T 0$. Values remained stable over the following 12 months. The SF-36 MCS shows a slightly different course. Values improved slower and showed significant improvement not until 24 months (T2) versus preoperative values (T0); see Figs. 4 and 5.

\section{Discussion}

The present study shows a significant pain reduction as well as improved QoL in surgically treated VO patients after 1 year. Improvement remained nearly constant over the 2-year observation time, but levels were markedly below QoL data published for general population. The 1-year mortality of $20 \%$ illustrates the severity and life-threatening nature of this clinical entity.

Although the optimal choice of therapy for this heterogeneous clinical entity cannot be uniformly determined, the goal of VO therapy is to cure the underlying infection to restore function and reduce pain. High-quality studies with information regarding the effectiveness of individual therapy options are lacking, especially considering that QoL is of particular importance for evaluating success, along with functional aspects $[6,20]$. Even though conservative treatment is the choice for the majority of the patients, it can be estimated that $20-40 \%$ of affected patients will undergo surgery [17].

The current study is the first prospective evaluation of QoL and the mortality rates of surgically treated $\mathrm{VO}$ patients over a 2-year period.

The older patients and patients with comorbidities are particularly susceptible to this disease. In this study, the average age was 69 years, with $66 \%$ of patients being male. A pathogen was identified in $71 \%$ of cases, the most 
Fig. 1 Flowchart for patient selection with additional QoL data

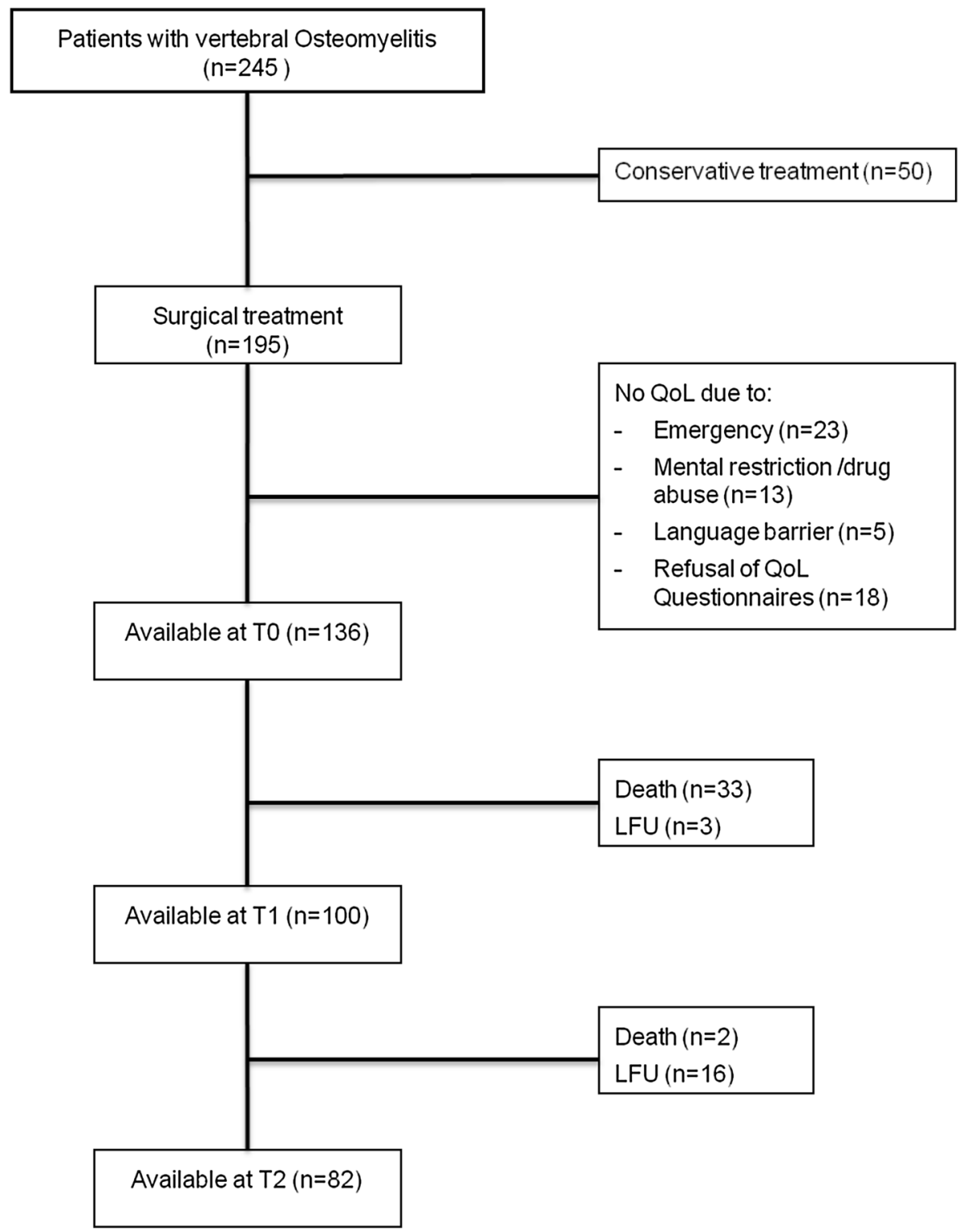

QoL:Quality of Life; T0: pre-operatively; T1: at 12 months; T2: at 24 months; LFU: Lost to follow-up common pathogen being Staphylococcus aureus (49\%). The lumbar spine was most often affected (56\%). Baseline characteristics in this study were typical and did not differ from other studies $[2,3,16]$. Average hospital inpatient stay of 30-49 days was reported in the literature and confirmed with our average stay of 33 days [10]. Recent retrospective evaluations of a Danish registry show the high mortality of this disease with rates of $20 \%$ and $21 \%$ in the first year after diagnosis $[5,9]$. These findings were also reproduced in our patient collective with a total mortality of $23 \%$ over a 2 -year follow-up. The course of our Kaplan-Meier curve mirrors Kehrer et al.'s hypothesis that VO shows a dramatic course especially in the first year because of its inflammatory nature in patients with multiple comorbidities [5]. Our study shows not only a high overall mortality but a high 1-year mortality with $20 \%$. The significantly lower mortality rate of $4 \%$ after the first year has also been confirmed by published reports. 
Table 1 Basic characteristics and clinical details of surgically treated vertebral osteomyelitis patients

\begin{tabular}{|c|c|}
\hline & $\begin{array}{l}\text { Surgically } \\
\text { treated } \\
(n=195)\end{array}$ \\
\hline \multicolumn{2}{|l|}{ Age } \\
\hline Median (IQR) & $69(51-87)$ \\
\hline \multicolumn{2}{|l|}{ Gender } \\
\hline Male, $n(\%)$ & $128(66)$ \\
\hline \multicolumn{2}{|l|}{ Spinal level } \\
\hline Cervical, $n(\%)$ & $5(3)$ \\
\hline Thoracic, $n(\%)$ & $46(24)$ \\
\hline Thoracolumbar, $n(\%)$ & $13(7)$ \\
\hline Lumbar, $n(\%)$ & $109(56)$ \\
\hline Multifocal, $n(\%)$ & $15(8)$ \\
\hline \multicolumn{2}{|l|}{ ASA } \\
\hline $1, n(\%)$ & $5(3)$ \\
\hline $2, n(\%)$ & $56(29)$ \\
\hline $3, n(\%)$ & $116(60)$ \\
\hline $4, n(\%)$ & $18(10)$ \\
\hline \multicolumn{2}{|l|}{ Recurrence } \\
\hline Yes, $n(\%)$ & $8(4)$ \\
\hline \multicolumn{2}{|l|}{ Microbiological diagnosis } \\
\hline Yes, $n(\%)$ & $138(71)$ \\
\hline Staphylococcus aureus, $n(\%)$ & $68(49)$ \\
\hline Coagulase neg. Staphylococci, $\mathrm{n}(\%)$ & $22(16)$ \\
\hline Enterococcus Species, $n(\%)$ & $7(5)$ \\
\hline Streptococcus Species, $n(\%)$ & $10(7)$ \\
\hline Gram-neg. pathogens, $n(\%)$ & $14(10)$ \\
\hline Anaerobes, $n(\%)$ & $8(5)$ \\
\hline Candida Species, $n(\%)$ & $3(2)$ \\
\hline Mycobateria Species (xenopi, bovis), $n(\%)$ & $6(4)$ \\
\hline \multicolumn{2}{|l|}{ Neurological deficits } \\
\hline Yes, $n(\%)$ & $57(29)$ \\
\hline \multicolumn{2}{|l|}{ Inpatient length of stay } \\
\hline Median (IQR) & $33(11-55)$ \\
\hline
\end{tabular}

$I Q R$ interquartile range

After the first year of disease onset, patients no longer die from the infection itself but from comorbidities [5, 8, 9, 21].

Retrospective studies have already suggested that the exclusive consideration of functional aspects (e.g., neurological deficits) in the treatment of VO is not sufficient to reflect the complexity of the disease. Further long-lasting negative effects such as pain and reduction in QoL even after healing should be considered [6]. The available studies on QoL are mostly retrospective and primarily concerned with QoL comparison in patients undergoing surgery versus conservatively treated patients or between various operative strategies. Looking at these studies, there is a trend toward increased patient satisfaction and quality of life with surgical versus conservative treatment $[12,13]$. In a comparison of operative versus conservative therapy for VO patients over 65, Sobottke et al. [12] found no significant differences in QoL scores of COMI, ODI, and SF-36. Nasto et al. [14] also compared both forms of therapy for $\mathrm{VO}$ (operative versus conservative) by using the QoL scores SF-12 and EQ-5D. They found increased satisfaction of patients who had undergone surgery, based on significantly higher QoL up to 6 months post-surgery. After another 3 months, this difference was no longer evident. The review article by Rutges et al. [20] points out that many fundamental aspects of VO therapy remain unclear because prospective long-term studies are lacking. They state that VO presents a dramatic clinical picture associated with high rates of orthopedic and neurological complications and significant worsening in QoL.

To the best of our knowledge, this is the first prospective study showing significant improvements in the QoL scores COMI, ODI, SF-36, and EQ-5D over the clinical course after successful surgical treatment of VO. The VAS values for back and/or leg pain were significantly reduced at 1 and 2 years compared to baseline values. In long-term follow-up (FU), i.e., 2 years after surgery, this was still observed for all parameters, although there was no significant difference between the first- and second-year values. A QoL plateau was reached that remained stable over the 2 years. However, despite the significant increase in QoL, values of the ODI, SF-36, and EQ-5D were below published values of both a general population and a chronic back pain population. These data coincide with the published retrospective reports. In 2008, O'Daly et al. [6] reported that the QoL of successfully treated VO patients was improved but remained significantly reduced compared to normal populations, regardless of whether they were treated conservatively or surgically.

In 2017, Dragsted et al. [17] first analyzed mortality and QoL using ODI and EQ-5D only in patients undergoing surgery. The 1-year mortality of $6.5 \%$ in that study is markedly less than the 1-year mortality in our study. The reason for this might be the lower average age of 60 years, but also the lower patient number $(n=65)$. This group also confirmed significantly reduced QoL for these patients compared to general population using the ODI and EQ-5D. All of the QoL data we collected showed significant improvements compared to baseline. VAS values for pain were significantly reduced. Our mean ODI values $(T 1=29$ and $T 2=29)$ 
Fig. 2 Kaplan-Meier survival plot of VO patients surgically treated

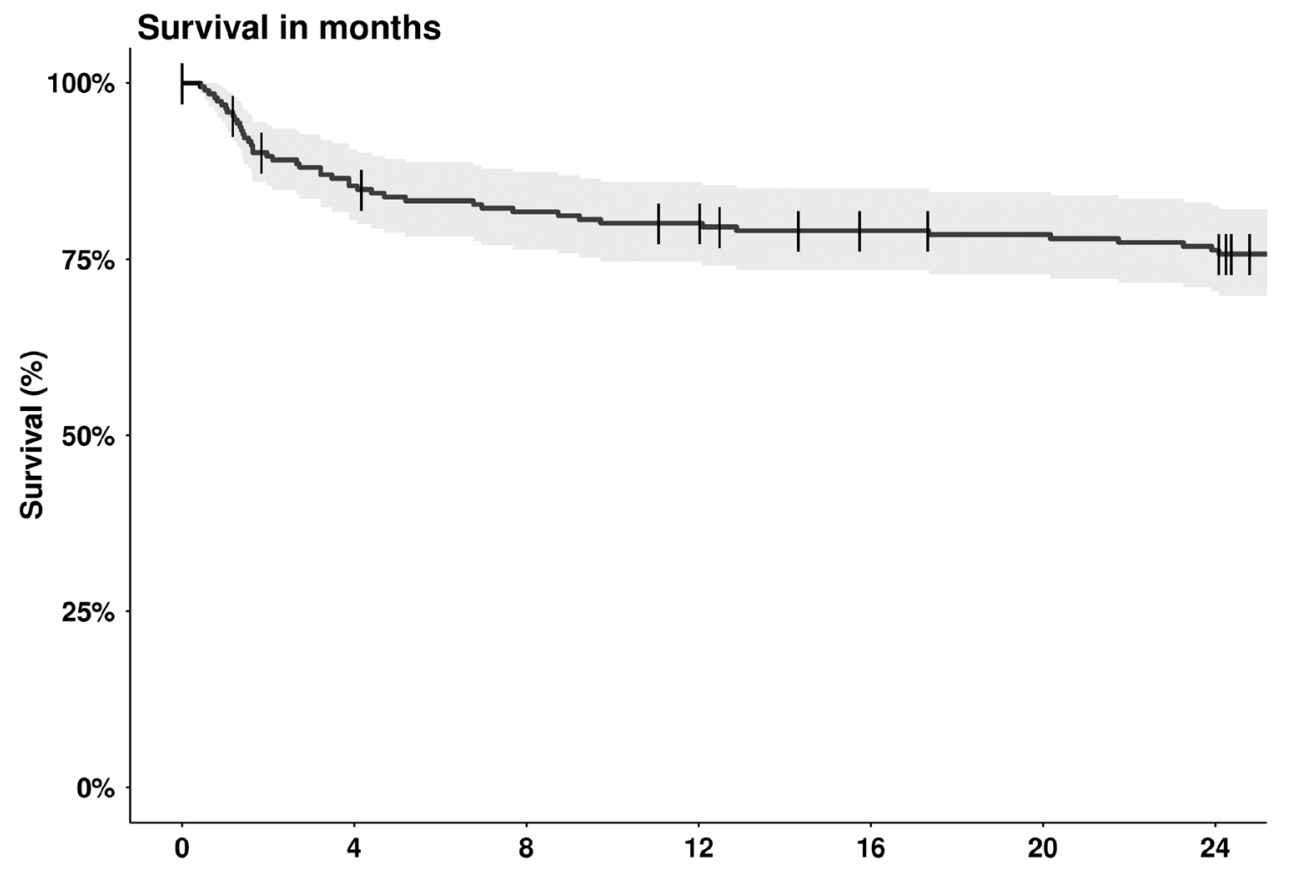

correspond to the retrospectively determined mean values in VO patients as reported in the literature [17]. They are above the values of Fairbank et al. [22] for healthy patients and above those of Tonosu et al. [23] with an age-standardized cutoff value $(\mathrm{ODI}=22$ ) for restrictive back pain. Comparing the mean values of MCS and PCS of the SF-36 with those for an age-matched general German population published by Bullinger et al. [24], the mean PCS value $(T 1=50$ and $T 2=45$ ) is not only below the comparative value of a healthy person $(\mathrm{PCS}=92$ ) but also well below that of a back pain patient $(\mathrm{PCS}=54)$. The mean MCS values $(T 1=43$ and $T 2=54$ ) were also well below the mean value of a healthy person (MCS $=71)$, and at 2 years it was comparable with
QoL of a back pain patient (MCS =55). Also, the EQ-5D index value for the German general population (0.9) lies above our mean EQ-5D index values $(T 1=0.6$ and $T 2=0.5)$ [25].

The strengths of the current study include a high number of patients, prospective design with a long FU over 2 years, and a "Lost to Follow-Up" (LFU) of only 9.7\%, which is low for a clinical study. In addition to the back pain-specific QoL score ODI, we included the established QoL scores of SF-36/EQ-5D. A VAS was used to achieve adequate pain assessment. The ODI has high validity for severe illnesses, the SF-36 for moderately severe illnesses, and the EQ-5D for mild illnesses [22, 26-28]. In our opinion, the use of 
Table 2 Pain and QoL scores at $T 0, T 1$ and $T 2$

\begin{tabular}{|c|c|c|}
\hline & Median (95\% CI) & $p$ value \\
\hline \multicolumn{3}{|c|}{ VAS back } \\
\hline$T 0$ & $8.2(7.8 ; 8.6)$ & Reference \\
\hline$T 1$ & $2.9(2.4 ; 3.5)$ & $<0.001$ \\
\hline$T 2$ & $3.1(2.4 ; 3.7)$ & $<0.001$ \\
\hline \multicolumn{3}{|c|}{ VAS leg } \\
\hline$T 0$ & $4.6(3.9 ; 5.2)$ & Reference \\
\hline$T 1$ & $2.0(1.5 ; 2.5)$ & $<0.001$ \\
\hline$T 2$ & $1.9(1.4 ; 2.6)$ & $<0.001$ \\
\hline \multicolumn{3}{|l|}{ ODI } \\
\hline$T 0$ & 75.7 (72.6; 78.9) & Reference \\
\hline$T 1$ & $29.1(24.4 ; 33.7)$ & $<0.001$ \\
\hline$T 2$ & $29.7(24.0 ; 35.1)$ & $<0.001$ \\
\hline \multicolumn{3}{|c|}{ SF-36 MCS } \\
\hline$T 0$ & $32.4(29.9 ; 34.9)$ & Reference \\
\hline$T 1$ & $43.5(34.8 ; 52.2)$ & 0.123 \\
\hline$T 2$ & $54.1(48.9 ; 59.4)$ & 0.018 \\
\hline \multicolumn{3}{|c|}{ SF-36 PCS } \\
\hline$T 0$ & $25.8(24.6 ; 27.1)$ & Reference \\
\hline$T 1$ & $49.7(42.9 ; 56.6)$ & 0.009 \\
\hline$T 2$ & $44.7(38.8 ; 50.6)$ & 0.004 \\
\hline \multicolumn{3}{|c|}{ COMI } \\
\hline$T 0$ & $8.8(8.6 ; 9.1)$ & Reference \\
\hline$T 1$ & $2.4(0.9 ; 3.7)$ & $<0.001$ \\
\hline$T 2$ & $2.0(0.8 ; 3.2)$ & $<0.001$ \\
\hline \multicolumn{3}{|c|}{ EQ-5D } \\
\hline$T 0$ & $-0.1(-0.2 ; 0.0)$ & Reference \\
\hline$T 1$ & $0.6(0.5 ; 0.7)$ & $<0.001$ \\
\hline$T 2$ & $0.5(0.3 ; 0.6)$ & $<0.001$ \\
\hline
\end{tabular}

VAS Visual Analogue Scale, ODI Oswestry Disability Index; $S F-36$ Short Form 36, MCS mental component score, PCS physical component score, COMI Core Outcome Measures Index, $T 0$ preoperatively, $T 1$ at 12 months, $T 2$ at 24 months these appropriate tools enabled the successful capture of the entire QoL spectrum for this very heterogeneous patient population.

Limitations of this study may be a possible selection bias, since this is a tertiary care facility primarily treating referred complicated cases from other hospitals. Due to the high mortality in the first year, data from the most severe patients are missing. More pre- and postoperative factors (e.g. severity of neurological deficit, comorbidities, duration of antibiotics, extent of vertebral destruction, etc.) need to be collected prospectively to perform a subgroup analysis of the QoL scores in patients after surgical treatment for VO.

\section{Conclusion}

Surgical treatment of VO patients leads to significantly improved QoL over the course of 2 years. Nevertheless, QoL levels of these patients were below those of the general population. Our results underscore the thesis that data focusing exclusively on cure, recurrence, and pain are underreporting the poor outcome of VO. Therefore, using spine disability questionnaires measuring QoL, such as ODI, SF-36, EQ-5D, and COMI, are mandatory to demonstrate comprehensively the severity of this clinical entity.

Since QoL levels remained nearly constant from $12(T 1)$ to 24 months (T2), a follow-up of 1 year might be sufficient for collecting VO outcome data.

Moreover, with a 2-year mortality rate of $23 \%$, our analysis confirms the high VO mortality and demonstrates the role of $\mathrm{VO}$ as a potentially life-threatening condition. 
Fig. 3 VAS back and leg at $T 0$, $T 1$ and $T 2$

Fig. 4 SF-36 MCS and PCS at $T 0, T 1$ and $T 2$

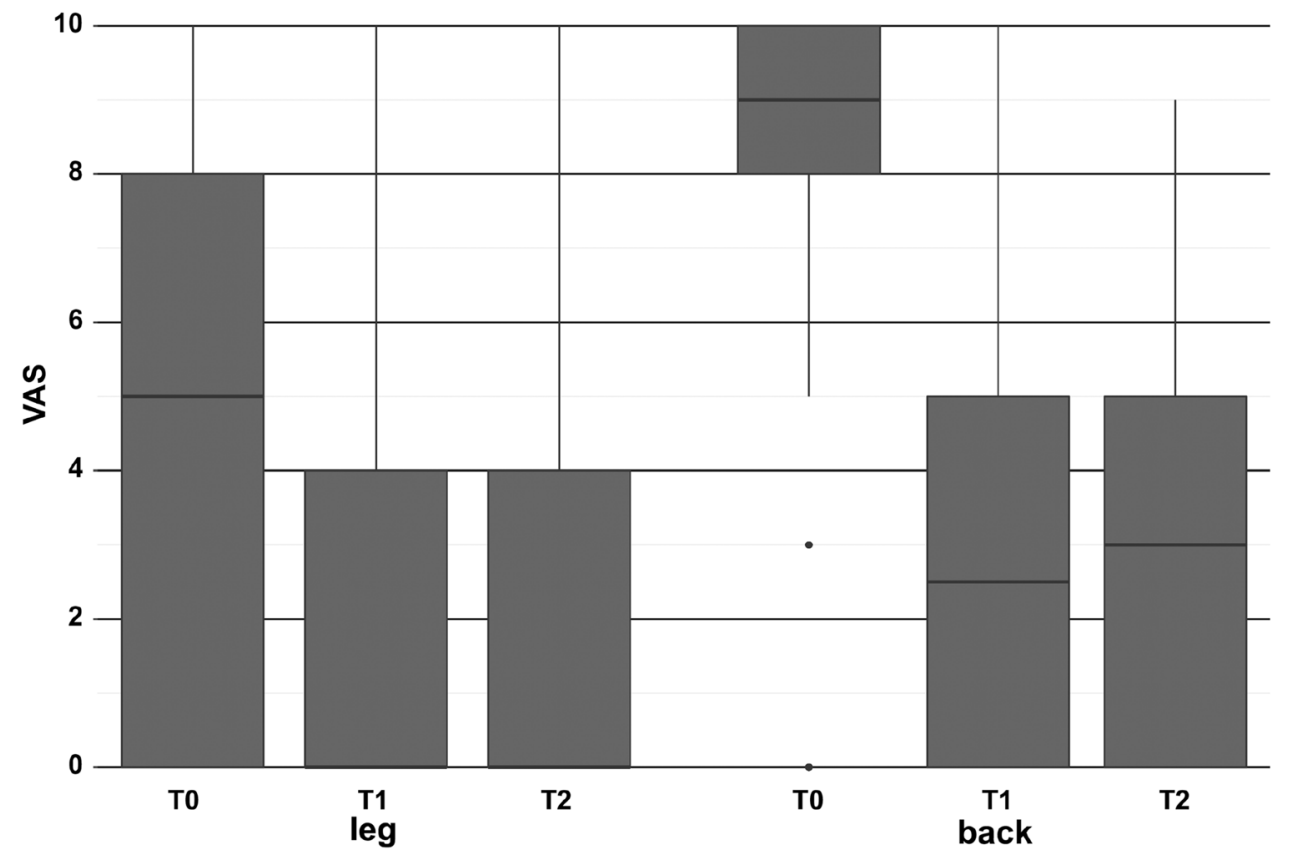

VAS: visual analogue scale; T0: pre-operatively; T1: at 12 months; T2: at 24 months

80

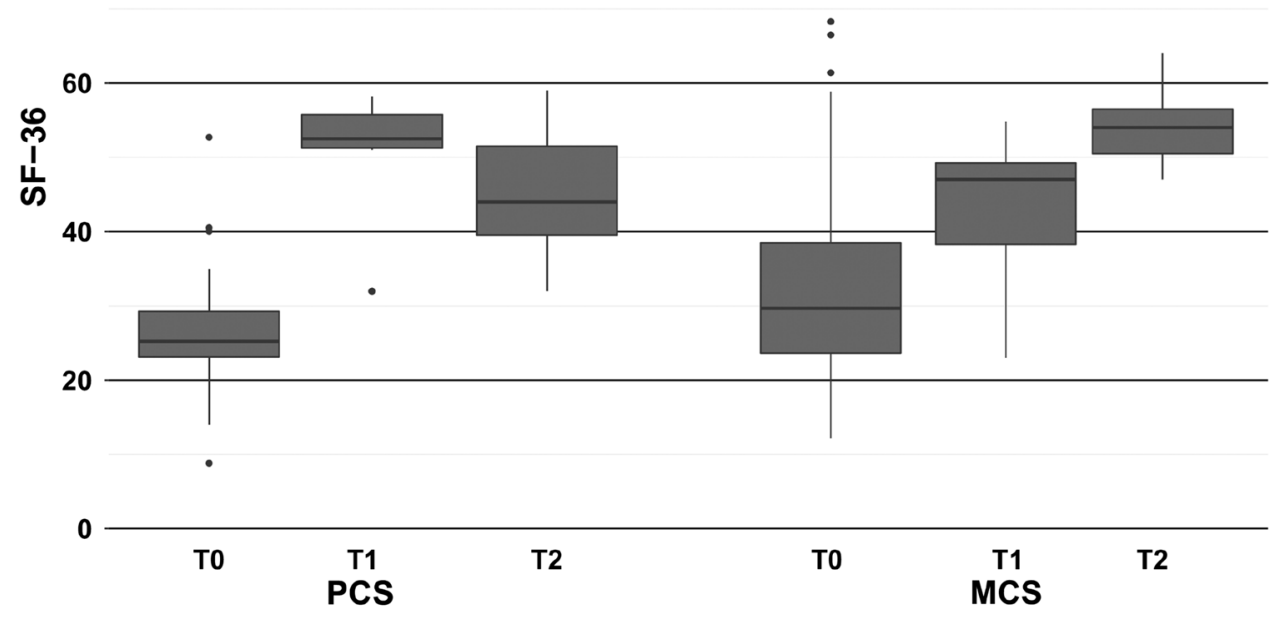

SF-36: Short Form 36; PCS: physical component score; MCS: mental component score; T0: preoperatively; T1: at 12 months; T2: at 24 months 
Fig. 5 ODI at $T 0, T 1$ and $T 2$

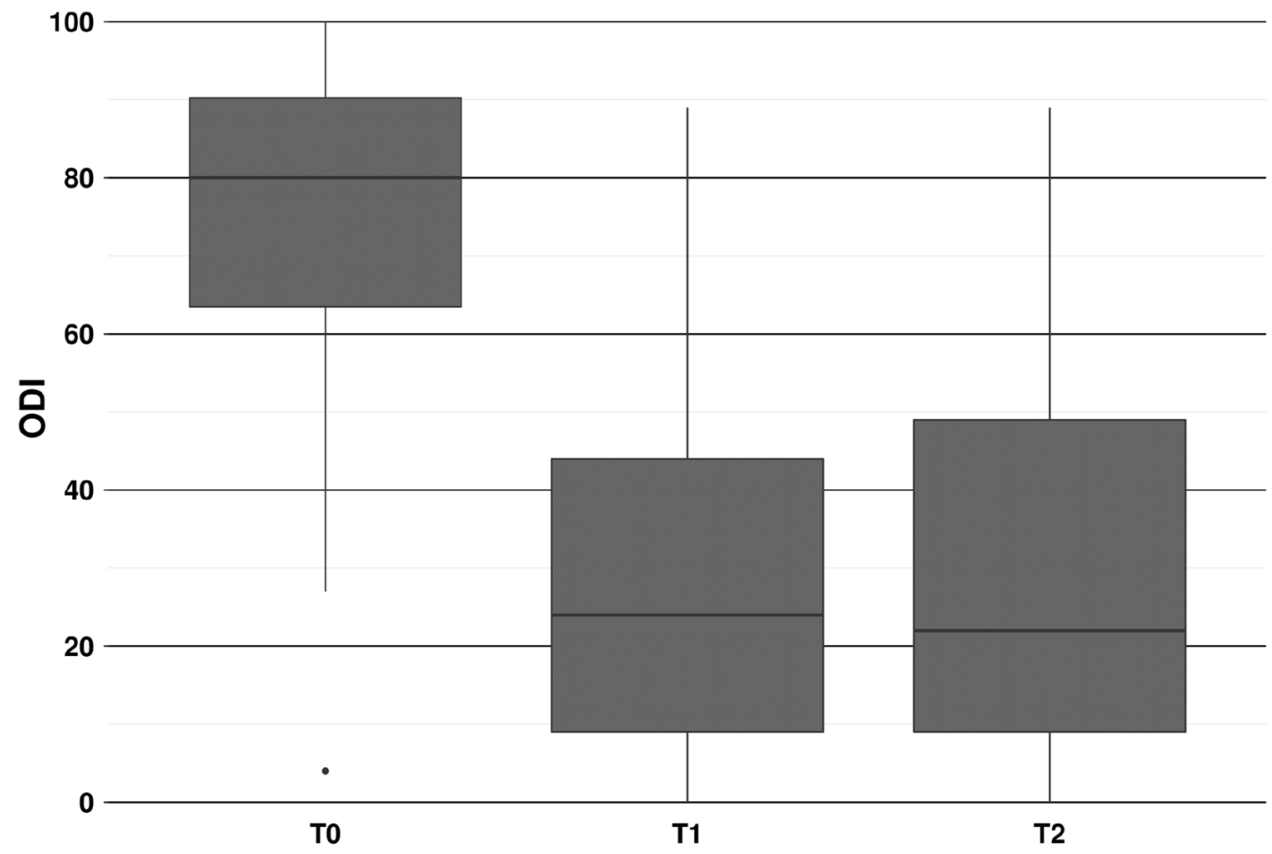

ODI: Oswestry Disability Score; TO: pre-operatively; T1: at 12 months; T2: at 24 months

Acknowledgements Open Access funding provided by Projekt DEAL. We would like to thank Katharina Braeger of IMSB, University of Cologne, for help with the graphics.

Author contributions AY, NJ, and JS planned the research design and wrote the manuscript. AY, KML, NJ, RS, and JS collected all data. Surgery was performed and evaluated by PE, RS, JS, MJS, KZ, and KS. NJ, COL, and AY discussed all infectious diseases counseling services. The entire data were controlled by NJ, AY, JS, RS, KML, and COL. Statistical analysis was made by KS and CS. The collected data and results were discussed with PE, JS, AY, and NJ. JS, $\mathrm{NJ}, \mathrm{COL}$, and $\mathrm{AY}$ helped by structuring and finalizing the manuscript. All authors have read and approved the final submitted manuscript.

Funding None.

\section{Compliance with ethical standards}

Conflict of interests The authors declare that they have no conflict of interest.

Availability of data and material The datasets used and/or analyzed during the current study are available from the corresponding author on reasonable request.

Code availability Software application or custom code: not applicable.

Consent to participate A written consent was available for all participants.
Ethics approval The study was approved by the ethics committee of our institution (file number: 09-182) and complies with the principles of the Declaration of Helsinki (1996).

Open Access This article is licensed under a Creative Commons Attribution 4.0 International License, which permits use, sharing, adaptation, distribution and reproduction in any medium or format, as long as you give appropriate credit to the original author(s) and the source, provide a link to the Creative Commons licence, and indicate if changes were made. The images or other third party material in this article are included in the article's Creative Commons licence, unless indicated otherwise in a credit line to the material. If material is not included in the article's Creative Commons licence and your intended use is not permitted by statutory regulation or exceeds the permitted use, you will need to obtain permission directly from the copyright holder. To view a copy of this licence, visit http://creativecommons.org/licenses/by/4.0/.

\section{References}

1. Zarghooni K, Rollinghoff M, Sobottke R, Eysel P (2012) Treatment of spondylodiscitis. Int Orthop 36:405-411

2. Nickerson EK, Sinha R (2016) Vertebral osteomyelitis in adults: an update. Br Med Bull 117:121-138

3. Herren C, Jung N, Pishnamaz M, Breuninger M, Siewe J, Sobottke R (2017) Spondylodiscitis: diagnosis and treatment options. Dtsch Arztebl Int 114:875-882

4. Pola E, Taccari F, Autore G et al (2018) Multidisciplinary management of pyogenic spondylodiscitis: epidemiological and clinical features, prognostic factors and long-term outcomes in 207 patients. Eur Spine J 27:229-236

5. Kehrer M, Pedersen C, Jensen TG, Hallas J, Lassen AT (2015) Increased short- and long-term mortality among patients with infectious spondylodiscitis compared with a reference population. Spine J 15:1233-1240 
6. O’Daly BJ, Morris SF, O'Rourke SK (2008) Long-term functional outcome in pyogenic spinal infection. Spine (Phila $\mathrm{Pa} 1976$ ) 33:E246-E253

7. Bundesamt Statistisches (2017) Diagnosedaten der Krankenhäuser ab 2000 (Eckdaten der vollstationären Patienten und Patientinnen). Gliederungsmerkmale, Jahre, Behandlungs/Wohnort

8. Aagaard T, Roed C, Dahl B, Obel N (2016) Long-term prognosis and causes of death after spondylodiscitis: a Danish nationwide cohort study. Infect Dis (Lond Engl) 48:201-208

9. Aagaard T, Roed C, Larsen AR et al (2014) Long-term mortality after Staphylococcus aureus spondylodiscitis: a Danish nationwide population-based cohort study. J Infect 69:252-258

10. Sobottke R, Seifert H, Fatkenheuer G, Schmidt M, Gossmann A, Eysel P (2008) Current diagnosis and treatment of spondylodiscitis. Dtsch Arztebl Int 105:181-187

11. Gupta A, Kowalski TJ, Osmon DR et al (2014) Long-term outcome of pyogenic vertebral osteomyelitis: a cohort study of 260 patients. Open Forum Infect Dis 1:ofu107

12. Sobottke R, Rollinghoff M, Zarghooni K et al (2010) Spondylodiscitis in the elderly patient: clinical mid-term results and quality of life. Arch Orthop Trauma Surg 130:1083-1091

13. Woertgen C, Rothoerl RD, Englert C, Neumann C (2006) Pyogenic spinal infections and outcome according to the 36-item short form health survey. J Neurosurg Spine 4:441-446

14. Nasto LA, Colangelo D, Mazzotta V et al (2014) Is posterior percutaneous screw-rod instrumentation a safe and effective alternative approach to TLSO rigid bracing for single-level pyogenic spondylodiscitis? Results of a retrospective cohort analysis. Spine J 14:1139-1146

15. Tsai TT, Yang SC, Niu CC et al (2017) Early surgery with antibiotics treatment had better clinical outcomes than antibiotics treatment alone in patients with pyogenic spondylodiscitis: a retrospective cohort study. BMC Musculoskelet Disord 18:175

16. Pola E, Autore G, Formica VM et al (2017) New classification for the treatment of pyogenic spondylodiscitis: validation study on a population of 250 patients with a follow-up of 2 years. Eur Spine J 26:479-488

17. Dragsted C, Aagaard T, Ohrt-Nissen S, Gehrchen M, Dahl B (2017) Mortality and health-related quality of life in patients surgically treated for spondylodiscitis. J Orthop Surg (Hong Kong) 25:2309499017716068

18. Gonzalvo A, Abdulla I, Riazi A, De La Harpe D (2011) Singlelevel/single-stage debridement and posterior instrumented fusion in the treatment of spontaneous pyogenic osteomyelitis/discitis: long-term functional outcome and health-related quality of life. J Spinal Disord Tech 24:110-115

19. von der Hoeh NH, Voelker A, Hofmann A et al (2018) Pyogenic spondylodiscitis of the thoracic spine: outcome of 1-stage posterior versus 2-stage posterior and anterior spinal reconstruction in adults. World Neurosurg 120:e297-e303

20. Rutges JP, Kempen DH, van Dijk M, Oner FC (2016) Outcome of conservative and surgical treatment of pyogenic spondylodiscitis: a systematic literature review. Eur Spine J 25:983-999

21. Kehrer M, Hallas J, Baelum J, Jensen TG, Pedersen C, Lassen AT (2017) Reduced ability to work both before and after infectious spondylodiscitis in working-age patients. Infect Dis (Lond, Engl) 49:95-103

22. Fairbank JC, Pynsent PB (2000) The Oswestry Disability Index. Spine (Phila Pa 1976) 25:2940-2952 (discussion 52)

23. Tonosu J, Takeshita K, Hara N et al (2012) The normative score and the cut-off value of the Oswestry Disability Index (ODI). Eur Spine J 21:1596-1602

24. Bullinger M (1995) German translation and psychometric testing of the SF-36 health survey: preliminary results from the IQOLA project. International quality of life assessment. Soc Sci Med 41:1359-1366

25. Szende AJB, Cabases J (2014) Self-reported population health: an international perspective based in EQ-5D. Springer, Berlin

26. DeVine J, Norvell DC, Ecker E et al (2011) Evaluating the correlation and responsiveness of patient-reported pain with function and quality-of-life outcomes after spine surgery. Spine (Phila Pa 1976) 36:S69-S74

27. Brettschneider C, Konig HH, Herzog W, Kaufmann C, Schaefert R, Konnopka A (2013) Validity and responsiveness of the EQ-5D in assessing and valuing health status in patients with somatoform disorders. Health Qual Life Outcomes 11:3

28. Johnsen LG, Hellum C, Nygaard OP et al (2013) Comparison of the SF6D, the EQ5D, and the oswestry disability index in patients with chronic low back pain and degenerative disc disease. BMC Musculoskelet Disord 14:148

Publisher's Note Springer Nature remains neutral with regard to jurisdictional claims in published maps and institutional affiliations. 


\section{Affiliations}

\section{Ayla Yagdiran ${ }^{1}$ (1) $\cdot$ Christina Otto-Lambertz ${ }^{1} \cdot$ Kristina Michaela Lingscheid $^{1} \cdot$ Krishnan Sircar $^{1}$ (D) $\cdot$ Christina Samel $^{2}$.

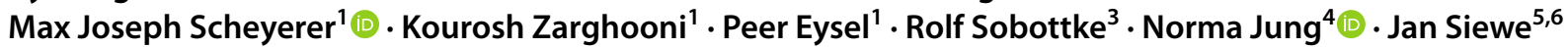

Christina Otto-Lambertz

christina.otto-lambertz@uk-koeln.de

Kristina Michaela Lingscheid

k.lingscheid@gmail.com

Krishnan Sircar

krishnan.sircar@uk-koeln.de

Christina Samel

christina.samel@uk-koeln.de

Max Joseph Scheyerer

max.scheyerer@uk-koeln.de

Kourosh Zarghooni

kourosh.zarghooni@uk-koeln.de

Peer Eysel

peer.eysel@uk-koeln.de

Rolf Sobottke

rolf.sobottke@rheinmaasklinikum.de

Norma Jung

norma.jung@uk-koeln.de
Jan Siewe

jan.siewe@klinikum-lev.de

1 Department of Orthopedic and Trauma Surgery, Faculty of Medicine and University Hospital Cologne, University of Cologne, Kerpener Str. 62, 50937 Cologne, Germany

2 Faculty of Medicine and University Hospital Cologne, Institute of Medical Statistics and Computational Biology, University of Cologne, Cologne, Germany

3 Department for Spine Surgery, Neurosurgery and Orthopedics, Rhein-Maas Klinikum GmbH, Mauerfeldchen 25, 52146 Würselen, Germany

4 Division of Infectious Diseases, Department I of Internal Medicine, Faculty of Medicine and University Hospital Cologne, University of Cologne, Cologne, Germany

5 Department for Spine Surgery, Klinikum Leverkusen gGmbH, Am Gesundheitspark 11, 51375 Leverkusen, Germany

6 Faculty of Medicine, University Hospital, Cologne, Germany 